\title{
The response of superpressure balloons to gravity wave motions
}

\author{
R. A. Vincent ${ }^{1}$ and A. Hertzog ${ }^{2}$ \\ ${ }^{1}$ Physics Department, University of Adelaide, Adelaide 5005, Australia \\ ${ }^{2}$ Laboratoire de Meteorologie Dynamique, École Polytechnique, Palaiseau, France \\ Correspondence to: R. A. Vincent (robert.vincent@adelaide.edu.au) \\ Received: 2 December 2013 - Published in Atmos. Meas. Tech. Discuss.: 13 December 2013 \\ Revised: 13 February 2014 - Accepted: 5 March 2014 - Published: 23 April 2014
}

\begin{abstract}
Superpressure balloons (SPB), which float on constant density (isopycnic) surfaces, provide a unique way of measuring the properties of atmospheric gravity waves (GW) as a function of wave intrinsic frequency. Here we devise a quasi-analytic method of investigating the SPB response to GW motions. It is shown that the results agree well with more rigorous numerical simulations of balloon motions and provide a better understanding of the response of SPB to $\mathrm{GW}$, especially at high frequencies. The methodology is applied to ascertain the accuracy of GW studies using $12 \mathrm{~m}$ diameter SPB deployed in the 2010 Concordiasi campaign in the Antarctic. In comparison with the situation in earlier campaigns, the vertical displacements of the SPB were measured directly using GPS. It is shown using a large number of Monte Carlo-type simulations with realistic instrumental noise that important wave parameters, such as momentum flux, phase speed and wavelengths, can be retrieved with good accuracy from SPB observations for intrinsic wave periods greater than ca. $10 \mathrm{~min}$. The noise floor for momentum flux is estimated to be ca. $10^{-4} \mathrm{mPa}$.
\end{abstract}

\section{Introduction}

Superpressure balloons (SPB) have been used in both the troposphere and lower stratosphere since the early 1960s (TWERLE Team, 1977). The balloons use closed, inextensible, spherical envelopes filled with a fixed amount of gas. After launch, balloons ascend until they reach a float level where atmospheric density matches the balloon density. On this isopycnic or equilibrium density surface (EDS) a balloon is free to float horizontally with the motion of the wind. Hence, SPB behave as quasi-Lagrangian tracers in the atmosphere.
Tracking the horizontal position of SPB using global positioning satellite (GPS) techniques means that SPB are well suited to study horizontal motions in the atmosphere. Measurement of vertical air motions is, however, more difficult because of the small vertical displacements that SPB generally undergo. A balloon displaced from its EDS experiences buoyancy forces that act to restore it, so it undergoes neutral buoyancy oscillations (NBO) around its EDS. Furthermore, the EDS itself will oscillate in the presence of gravity (buoyancy) waves (GW). By analysing the governing equation of motion through numerical integration, Massman (1978) explored the nature of both these factors, including the amplitude and phase response of an SPB to GW-induced sinusoidal variations of the EDS. Nastrom (1980) extended this work by considering the simultaneous wave-induced variations of density and vertical wind. He developed an analytical relationship between the amplitude and phase of a SPB in the presence of a sinusoidal gravity wave. Massman (1981) demonstrated how SPB can be used to study gravity wave activity in the Southern Hemisphere upper troposphere and lower stratosphere. An advantage of using SPB to study gravity waves is that, because the balloons drift with the background wind, they measure the intrinsic frequency (frequency relative to a moving reference frame). It is the intrinsic frequency that appears naturally in the Navier-Stokes equations that determine important wave properties. In contrast to either ground- or space-based sensors, SPB observations have the ability both to fully characterize wave packets and to provide such information over wide geographic regions (Alexander et al., 2010).

The French Space Agency, CNES, developed and applied $8.5 \mathrm{~m}$ and $10 \mathrm{~m}$ diameter SPB and, more recently, developed $12 \mathrm{~m}$ diameter balloons that can carry payloads of up to $40 \mathrm{~kg}$. These balloons are significantly larger than those 
used in previous studies and have long flight times on the order of months. A mixture of 8 and $10 \mathrm{~m}$ diameter SPB were used to study motions and transport in the Antarctic stratosphere during the Stratéole/Vorcore campaign in 2005 (Hertzog et al., 2007). The long duration of SPB flights during Vorcore proved invaluable in studies of atmospheric gravity waves and the geographical variation of wave sources (Vincent et al., 2007; Boccara et al., 2008; Hertzog et al., 2008; Walterscheid et al., 2012). In the subsequent Concordiasi campaign in 2010, held during the Antarctic late winter and spring, $12 \mathrm{~m}$ diameter SPB were used exclusively (Rabier et al., 2010).

A limitation of the Vorcore observations of gravity waves by SPB was the effective 15 min sampling interval imposed by the data transmission rate. The corresponding Nyquist period of about $30 \mathrm{~min}$ was considerably longer than the approximately $5 \mathrm{~min}$ short period cutoff to the gravity wave spectrum due to the Brunt-Väisälä frequency in the lower stratosphere. In subsequent SPB campaigns this limitation was overcome by the implementation of a new communications system which allows a time resolution of about $30 \mathrm{~s}$. Improved time resolution is particularly important for SPB studies in the tropics where convection is predicted to generate waves over a wide range of scales and periods, but with wavelengths between 5 and $50 \mathrm{~km}$ and periods between 10 and 60 min being especially prominent (Piani et al., 2000; Beres, 2004; Lane and Moncrieff, 2008; Jewtoukoff et al., 2013). Hence, SPB observations now cover the full range of the GW spectrum, a unique characteristic of this technique (Preusse et al., 2008; Alexander et al., 2010).

This paper consists of two parts. In the first part we investigate the response of SPB to gravity wave motions by extending the analysis of Nastrom (1980) of the balloon equation of motion. We introduce a quasi-analytic method for analyzing the SPB response to an atmospheric wave. When an SPB responds to a gravity-wave-induced displacement of the EDS the equation of motion is such that there is a phase shift between the balloon and the EDS displacement. This phase shift is a factor in the retrieval of important GW parameters, including the intrinsic phase speed (i.e., the speed relative to the background wind). Amplitudes and phases derived from the simplified technique are compared with the numerical calculations of the equation of motion of the SPB and it is shown that they agree well. There is a specific emphasis on the response of the newer $12 \mathrm{~m} \mathrm{SPB}$, although the results are quite applicable to the smaller diameter balloons. In the second part of the paper, we test how well the improved instrumentation on the $12 \mathrm{~m} \mathrm{SPB}$ is able to detect GW motions and retrieve wave parameters. For this aspect we carried out a large number of statistical realizations that covered the full spectrum of GW frequencies. The computational efficiency of the analytic technique means that it is very suitable for this analysis. This second part extends the work of Boccara et al. (2008) who dealt with Vorcore observations, and in particular only considered the case of hydrostatic waves.

\section{Theory}

Following Nastrom (1980), the governing equation of motion in the vertical direction for a balloon floating in the atmosphere is

$$
\begin{gathered}
\left(M_{\mathrm{B}}+\eta M_{\mathrm{a}}\right) \frac{\partial^{2} \zeta_{b}^{\prime}}{\partial t^{2}}=-g\left(M_{\mathrm{B}}-M_{\mathrm{a}}\right)-\frac{1}{2} \rho_{\mathrm{a}} C_{\mathrm{d}} A_{\mathrm{B}} \\
\left(\frac{\partial \zeta_{b}^{\prime}}{\partial t}-w^{\prime}\right)\left|\frac{\partial \zeta_{b}^{\prime}}{\partial t}-w^{\prime}\right|+\left(M_{\mathrm{B}}+\eta M_{\mathrm{a}}\right) \frac{\partial w^{\prime}}{\partial t}
\end{gathered}
$$

where the symbols are defined in Table 1.

Physically, the terms on the right hand side of Eq. (1) can be attributed to the three non-negligible forces acting on the balloon. The first term is the buoyancy force, which acts whenever the balloon is displaced vertically to restore it to its EDS. The second term is the drag force, which acts to resist the motion of the balloon. The third term comes from a dynamic force supplied to the balloon by the surrounding atmosphere when it is in motion. Any other forces acting on the balloon, such as skin friction drag, aerodynamic lift and small-scale turbulence are assumed to be small in comparison (Nastrom, 1980). The left hand side of the equation is then the net force acting on the balloon.

Assuming small vertical displacements and considering spherical SPBs, Eq. (1) can be simplified to

$$
\begin{aligned}
\frac{\partial^{2} \zeta_{b}^{\prime}}{\partial t^{2}}= & -\omega_{\mathrm{B}}^{2} \zeta_{b}^{\prime}+\frac{2}{3} g R-A\left(\frac{\partial \zeta_{b}^{\prime}}{\partial t}-w^{\prime}\right) \\
& \left|\frac{\partial \zeta_{b}^{\prime}}{\partial t}-w^{\prime}\right|+\frac{\partial w^{\prime}}{\partial t},
\end{aligned}
$$

where $R$ is a wave-induced relative density perturbation and $A$ is a constant dependent on balloon parameters (Nastrom, 1980). The neutral buoyancy oscillation (NBO) frequency $\omega_{\mathrm{B}}$ is the frequency with which a constant-volume balloon will oscillate around its EDS, and is given by

$\omega_{\mathrm{B}}^{2}=\frac{2 g}{3 T}\left(\frac{\partial T}{\partial z}+\frac{g}{R_{\mathrm{a}}}\right)$

with temperature $T$, vertical temperature gradient $\partial T / \partial z$ and atmospheric gas constant $R_{\mathrm{a}}$. A balloon of radius $r$ and drag coefficient $C_{\mathrm{d}}$ gives $A$ as

$A=\frac{C_{\mathrm{d}}}{4 r}$.

The first two terms of Eq. (2) originate from the buoyancy term and the third and fourth terms come from the drag and dynamic terms, respectively. This simplification assumes that the balloon is always near to its EDS, so that $M_{\mathrm{a}} \approx M_{\mathrm{B}}$ at all times. It is also assumed that the balloon is perfectly spherical. See Nastrom (1980) for further details.

If the EDS is disturbed by a GW of intrinsic frequency $\hat{\omega}$ and vertical velocity amplitude $w_{o}$, so that the instantaneous 
Table 1. Definition of terms used in the text.

\begin{tabular}{ll}
\hline Symbol & Definition \\
\hline$M_{\mathrm{B}}$ & Mass of balloon system \\
$M_{\mathrm{a}}$ & Mass of the air displaced by the balloon system \\
$\eta$ & Added mass coefficient (1/2 for a sphere in a perfect fluid) \\
$\zeta_{b}^{\prime}$ & Vertical displacement the balloon from its EDS \\
$\rho_{\mathrm{a}}$ & Density of ambient atmosphere \\
$C_{\mathrm{d}}$ & Drag coefficient \\
$A_{\mathrm{B}}$ & Cross-sectional area of balloon \\
$w^{\prime}$ & Vertical wind velocity. \\
\hline
\end{tabular}

vertical velocity is $w^{\prime}=w_{o} e^{-i \hat{\omega} t}$, then the wave-induced fractional density perturbation is given by the polarization relation (e.g., Hines, 1960) as

$R=\frac{\rho^{\prime}}{\bar{\rho}}=i \frac{N^{2}}{g \hat{\omega}} w^{\prime}$,

where $\bar{\rho}$ is the ambient density, $N$ is the Brunt-Väisälä frequency, defined as

$N^{2}=\frac{g}{T}\left(\frac{g}{c_{p}}+\frac{\partial T}{\partial z}\right)$

and $c_{p}$ is specific heat capacity.

High vertical resolution temperature soundings show that on global and seasonal scales $N^{2}$ ranges from $\sim 4 \times 10^{-4}$ to $\sim 8 \times 10^{-4} \mathrm{rad}^{2} \mathrm{~s}^{-2}$ at heights near $20 \mathrm{~km}$ (Grise et al., 2010). These values correspond to temperature gradients ranging between $\sim 0$ and $\sim 7 \mathrm{~K} \mathrm{~km}^{-1}$, with the largest values associated with the region just above the tropical tropopause. This means that for realistic temperature gradients, $\omega_{\mathrm{B}}$ is always greater than $N$; a gradient greater than $40 \mathrm{~K} \mathrm{~km}^{-1}$ is required for $\omega_{\mathrm{B}} / N<1$. Hence, at lower stratosphere heights, neutral buoyancy oscillations are always higher in frequency than the highest frequency gravity waves.

\section{Models}

\subsection{Numerical model}

For a GW of given intrinsic frequency and amplitude, Eq. (2) can be solved numerically to derive $\zeta_{b}^{\prime}$ as a function of time. As an example, consider a case study where the balloon parameters are typical of a $12 \mathrm{~m}$ diameter SPB used during the Concordiasi campaign. It is assumed that the atmospheric conditions used are similar to those experienced in the Antarctic lower stratosphere in early spring. Table 2 gives the basic atmospheric and balloon parameters. For the purposes of illustration, a gravity wave was used with a vertical wind perturbation amplitude of $w_{o}=1 \mathrm{~m} \mathrm{~s}^{-1}$ and intrinsic period $\hat{\tau}=15 \mathrm{~min}$ or angular frequency of $\hat{\omega}=6.98 \times$ $10^{-3} \mathrm{rad} \mathrm{s}^{-1}$. This produces a fractional density perturbation of $5.85 \times 10^{-3}$.
Table 2. Atmospheric and balloon parameters in the Antarctic lower stratosphere.

\begin{tabular}{ll}
\hline Parameter & Value \\
\hline $\bar{T}$ & $200 \mathrm{~K}$ \\
$\mathrm{~d} \bar{T} / \mathrm{d} z$ & $-1.60 \times 10^{-3} \mathrm{~K} \mathrm{~m}^{-1}$ \\
$N^{2}$ & $4 \times 10^{-4} \mathrm{~s}^{-2}$ \\
$\omega_{\mathrm{B}}^{2}$ & $9.7 \times 10^{-4} \mathrm{~s}^{-2}$ \\
$\bar{\rho}$ & $0.105 \mathrm{~kg} \mathrm{~m}^{-3}$ \\
$\bar{p}$ & $6000 \mathrm{~Pa}$ \\
$C_{\mathrm{d}}$ & 0.5 \\
$r$ & $6.0 \mathrm{~m}$ \\
$A$ & $0.0208 \mathrm{~m}^{-1}$ \\
\hline
\end{tabular}

Figure 1a shows the result of numerically solving Eq. (2) using a fourth order Runge-Kutta method (Press et al., 1992). For this example the total duration of the time series was $12.5 \mathrm{~h}$ (i.e., 50 oscillations) and a time step of $1 \mathrm{~s}(0.1 \%$ of the period) was used, although the results are not particularly sensitive to the time step. Transients due to the initial conditions persisted for less than a cycle, so the results shown in Fig. 1a are the steady-state response. The red line represents the vertical position of the balloon plotted against time. The blue line represents the balloon displacement derived using an analytic method described below.

While the numerical solution is almost sinusoidal, it is noticeable that higher frequency components are also present. The power spectral analysis of the whole $12.5 \mathrm{~h}$ period, shown in Fig. 1b, illustrates the absence of even harmonics and the dominance of the first harmonic over the other odd harmonics. The third harmonic is approximately ten percent in magnitude of the first harmonic and the fifth harmonic less than five percent. Higher harmonics are less than one percent of the first harmonic. This result supports the analysis of Nastrom (1980) that shows that only odd harmonics are present in the vertical displacement, with the first harmonic dominating.

The harmonic content shown in Fig. 1b is typical of the response to short period waves. However, the amplitude of the harmonics decreases as the wave period increases. At a period of $30 \mathrm{~min}$ for example, the third harmonic has an amplitude of less than $3 \%$ of the fundamental.

\subsection{Analytic model}

The dominance of the first harmonic in the balloon response shown in Fig. 1b suggests that a linear relationship between $\zeta_{b}^{\prime}$ and $\zeta^{\prime}$ is a reasonable approximation to the balloon's response to a gravity wave. Hence we now consider the balloon and its environment as a quasi-linear system, treating the gravity wave as the input and the balloon response as the output signal of this system. Using linear system theory there will exist a transfer function (complex frequency response) 

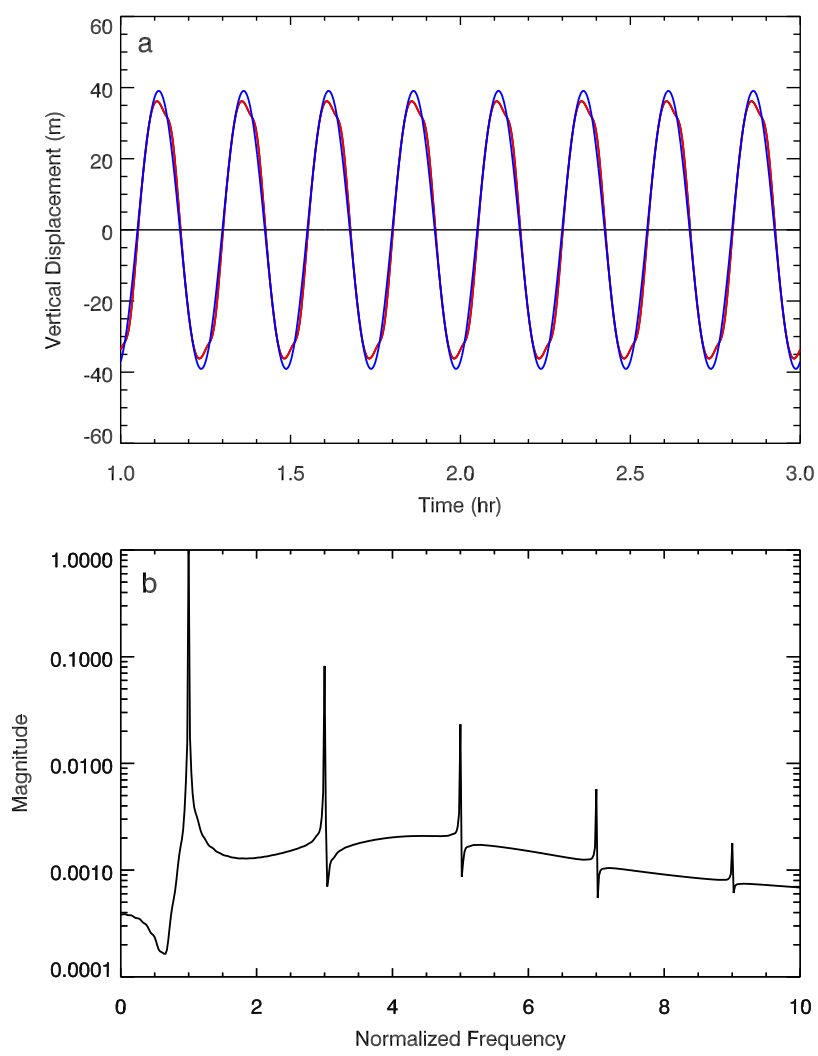

Fig. 1. (a) Vertical displacements of a $12 \mathrm{~m}$ diameter superpressure balloon induced by a gravity wave with period $15 \mathrm{~min}$ and vertical wind amplitude of $1 \mathrm{~m} \mathrm{~s}^{-1}$. Results are plotted for the interval 1 to $3 \mathrm{~h}$ after the simulation was turned on to avoid transient effects near $t=0$. The red curve shows the numerical simulation while the blue curve shows results from the analytic method. (b) Normalized power spectrum of the numerically simulated SPB vertical displacements computed from the whole $12.5 \mathrm{~h}$ duration of the time series.

relating the output to the input. The function is of the form:

$\frac{\zeta_{b}^{\prime}}{\zeta^{\prime}}=\underline{Z}=|\underline{Z}| e^{i \phi}$

where $|\underline{Z}|$ and $\phi$ are, respectively, the absolute value and phase of the transfer function $(\underline{Z}) ; \zeta_{b}^{\prime}$ is the vertical variation of the balloon around its EDS due to the gravity wave. Here the phase, $\phi$, is relative to the time of maximum wave displacement.

Now consider a sinusoidal GW for which the complex amplitude is $\zeta^{\prime}=\zeta_{o} e^{-i \hat{\omega} t}$, where $\zeta_{o}$ is the wave vertical displacement amplitude. The vertical wind and density perturbation in terms of $\zeta^{\prime}$ are, respectively,

$$
\begin{aligned}
& w^{\prime}=\frac{\partial \zeta^{\prime}}{\partial t}=-i \hat{\omega} \zeta_{o} e^{-i \hat{\omega} t}=-i \hat{\omega} \zeta^{\prime}, \\
& R=\frac{N^{2}}{g} \zeta^{\prime}
\end{aligned}
$$

It should be noted that Eq. (9) is an approximation that needs to be modified for large vertical wavelength GWs, such as those that might be generated by deep convection (Eckermann et al., 1998). Eckermann et al. (1998) show Eq. (9) is accurate to within a few percent in amplitude and phase for vertical wavelengths less than $20 \mathrm{~km}$ and so we use the approximation in the following analysis.

Substituting Eq. (8) and Eq. (9) into Eq. (2) and evaluating the derivatives of $\zeta_{b}^{\prime}$ and $\zeta^{\prime}$ gives

$$
\begin{gathered}
-\hat{\omega}^{2} \zeta_{b}^{\prime}=-\omega_{\mathrm{B}}^{2} \zeta_{b}^{\prime}+\frac{2}{3} N^{2} \zeta^{\prime}-A\left(-i \hat{\omega} \zeta_{b}^{\prime}+i \hat{\omega} \zeta^{\prime}\right) \\
\left|-i \hat{\omega} \zeta_{b}^{\prime}+i \hat{\omega} \zeta^{\prime}\right|-\hat{\omega}^{2} \zeta^{\prime} .
\end{gathered}
$$

From Eq. (7) and substituting for $\zeta_{b}^{\prime}$ while retaining only the first harmonic in the non-linear drag term leads to

$\underline{Z}=\frac{\frac{2}{3} N^{2}-\hat{\omega}^{2}-i A \hat{\omega}^{2} \zeta_{o} Y}{\omega_{\mathrm{B}}^{2}-\hat{\omega}^{2}-i A \hat{\omega}^{2} \zeta_{o} Y}$

where $Y \equiv|1-\underline{Z}|$.

A value for $\underline{Z}$ can be calculated iteratively using an initial value of $Y=1 .|\underline{Z}|$ converges to a fractional difference of less than $10^{-4}$ within two or three iterations for $\hat{\omega}^{2} \ll N^{2}$ (i.e., periods greater than about $10 \mathrm{~min}$ ) and within six steps for $\hat{\omega} \sim N$. Hence, the SPB response to any gravity wave can be obtained using Eq. (7), as illustrated in Fig. 1a where the blue line is the analytic solution. It is evident that the analytic solution slightly overestimates the numerical solution, but the difference is no more than a few meters.

\section{Analysis}

Further insights into the response of large diameter SPB to wave-induced motions are gained by considering both the numerical and analytic approaches. Here we use the same balloon parameters and atmospheric conditions as given in Sect. 3 and derive the response as a function of a number of gravity wave parameters.

Firstly, the value of $w_{o}$ was varied over a range from 0.1 to $2.0 \mathrm{~m} \mathrm{~s}^{-1}$ for the three different $\mathrm{GW}$ intrinsic periods of $\tau=15,30$ and $60 \mathrm{~min}$. Figure 2 shows the amplitude ratio, $|\underline{Z}|$, and phase computed using both the numerical and analytic methods. For all three periods the two methods give amplitude ratios that do not differ by more than $5 \%$ and phases that differ by no more than a few degrees.

Similar results are displayed in Fig. 3. Here the amplitude and phase response derived from the numerical and analytic methods are plotted as a function of wave period for values of $w_{o}$ that were fixed at values $0.5,1.0$ and $1.5 \mathrm{~m} \mathrm{~s}^{-1}$. The amplitude and phase start to vary markedly as the wave period approaches the buoyancy period of about $5 \mathrm{~min}$. Nevertheless, the relative amplitudes derived by the two methods agree well. Similarly, the phases agree to within at least $5^{\circ}$. We note that the expression that relates the density to vertical 


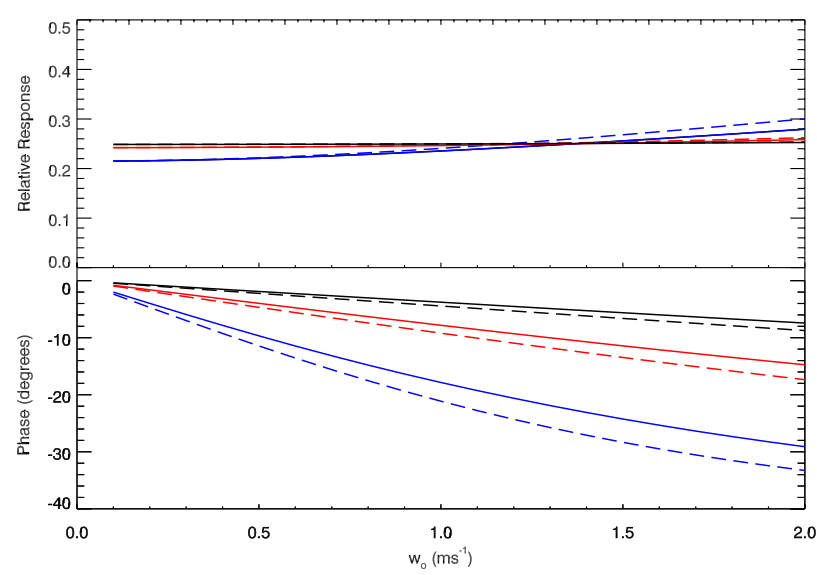

Fig. 2. Comparison of the analytical solution (dashed) with the numerical solution (solid) for the response of a $12 \mathrm{~m}$ diameter SPB to gravity waves with different values of vertical velocity amplitude and periods of $15 \mathrm{~min}$ (blue), $30 \mathrm{~min}$ (red) and $60 \mathrm{~min}$ (black). The top panel shows the balloon displacement normalized to wave displacement $\left(\zeta_{o}^{\prime} / \zeta_{o}\right)$ and the bottom panel the relative phase, $\phi$.

velocity perturbations, (Eq. 9), does not apply where $\hat{\omega}>N$, which is outside the internal gravity wave range. However, we retain it for the purposes of illustration of $\underline{Z}$. For example, arbitrarily setting $R=0$ when $\hat{\omega}>N$ gives almost identical curves to those shown in Fig. 3 with discontinuities at $\hat{\omega}=N$. In all subsequent analysis and discussion we are concerned only with internal waves in the range $N>\hat{\omega}>|f|$.

The analytic model also works for wave packets. Figure 4 shows the numerical and analytic solutions for a wave packet of a wave with frequency $\hat{\omega}$ and a Gaussian envelope defined as

$\zeta=\zeta_{o} e^{-t^{2} / 2 t_{g}^{2}} \cos (\hat{\omega} t)$

Here, the wave period is $\tau=15 \mathrm{~min}$ and the "width" parameter $t_{g}=\tau$, so the packet has about five oscillations. Again, the numerical response shows some influence of the odd harmonics, as demonstrated in the lower panel of Fig. 4, but otherwise there is good agreement between both solutions.

One benefit of the analytic approach is that it gives insight into the SPB response as a function of wave frequency. For example, when $\hat{\omega}^{2} \ll N^{2}$ it is evident from Eq. (11) that $|\underline{Z}| \rightarrow 2 N^{2} / 3 \omega_{\mathrm{B}}^{2} \equiv|Z|_{\text {EDS. This limiting value, when }}$ the balloon is on its EDS, has a numerical value of $|Z|_{\text {EDS }} \sim$ 0.25 with the temperature gradient used here. Similarly, the phase limit is $\phi \rightarrow 0$. These are the limiting values evident in Figs. 2 and 3 and they correspond to the behavior of a perfect isopycnic balloon. The actual value of $Z_{\text {EDS }}$ will depend on the ambient conditions, especially the temperature gradient as this determines $\omega_{\mathrm{B}}^{2}$ and $N^{2}$. $Z_{\mathrm{EDS}}$ is always less than 0.5 using realistic gradients in the lower stratosphere, as discussed at the end of Sect. 2.

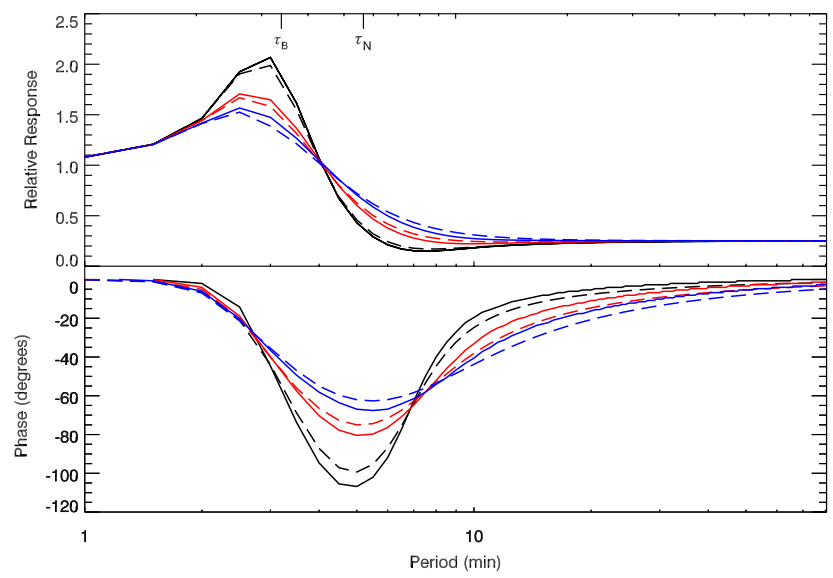

Fig. 3. As in Fig. 2, but with the SPB response as a function of wave period for waves with vertical velocity amplitudes of $w_{o}$ equal to 0.5 (black), 1.0 (red) and $1.5 \mathrm{~m} \mathrm{~s}^{-1}$ (blue). The symbols $\tau_{\mathrm{B}}$ and $\tau_{N}$ indicate the periods corresponding to the neutral buoyancy oscillation $\left(\omega_{\mathrm{B}}\right)$ and Brunt-Väisälä $(N)$ frequencies, respectively.

Manipulating Eq. (11) shows that

$\tan \phi=\frac{\left(\frac{2}{3} N^{2}-\omega_{\mathrm{B}}^{2}\right) A \hat{\omega}^{2} \zeta_{o} Y}{\left(\frac{2}{3} N^{2}-\hat{\omega}^{2}\right)\left(\omega_{\mathrm{B}}^{2}-\hat{\omega}^{2}\right)+\left(A \hat{\omega}^{2} \zeta_{o} Y\right)^{2}}$.

Since $2 / 3 N^{2}<\omega_{\mathrm{B}}^{2}$, the numerator in Eq. (13) is negative. The denominator is always positive, which means that $\phi$ is always negative and the balloon displacement lags the wave displacement. These results show that a SPB starts to depart substantially from its EDS for wave periods less than about $10 \min$ (i.e., for $\hat{\omega} \gtrsim N / 2$ ).

Two approximations give further insight into balloon behavior. First, in the low frequency limit when $\hat{\omega}^{2} \ll N^{2}$

$\tan \phi \rightarrow \frac{\left(\frac{2}{3} N^{2}-\omega_{\mathrm{B}}^{2}\right) A \hat{\omega}^{2} \zeta_{o} Y}{\frac{2}{3} N^{2} \omega_{\mathrm{B}}^{2}}$,

which shows that the phase is proportional to $\hat{\omega}^{2} \zeta_{o}=\hat{\omega} w_{o}$, all other parameters remaining constant. Hence the phase departures become greater for larger wave amplitudes and shorter periods, as seen in Fig. 2.

Second, when $\hat{\omega} \sim N$, then

$\tan \phi \approx \frac{\left(\frac{2}{3} N^{2}-\omega_{\mathrm{B}}^{2}\right)}{A \hat{\omega}^{2} \zeta_{o} Y}$,

so now the phase departure is greater for smaller amplitude waves, as observed in Fig. 3.

Equations (14) and (15) also show that $\tan \phi \propto A \propto r^{-1}$ when $\hat{\omega}^{2}<<N^{2}$ and $\tan \phi \propto A^{-1} \propto r$ when $\hat{\omega} \sim N$. So at lower frequencies the phase shifts will be greater for smaller balloons for a given wave amplitude, while the opposite is true when the wave frequency is near $N$. Finally, without going into details, it is straightforward to show that $|\underline{Z}| \propto r$ for $\hat{\omega} \sim N$. 

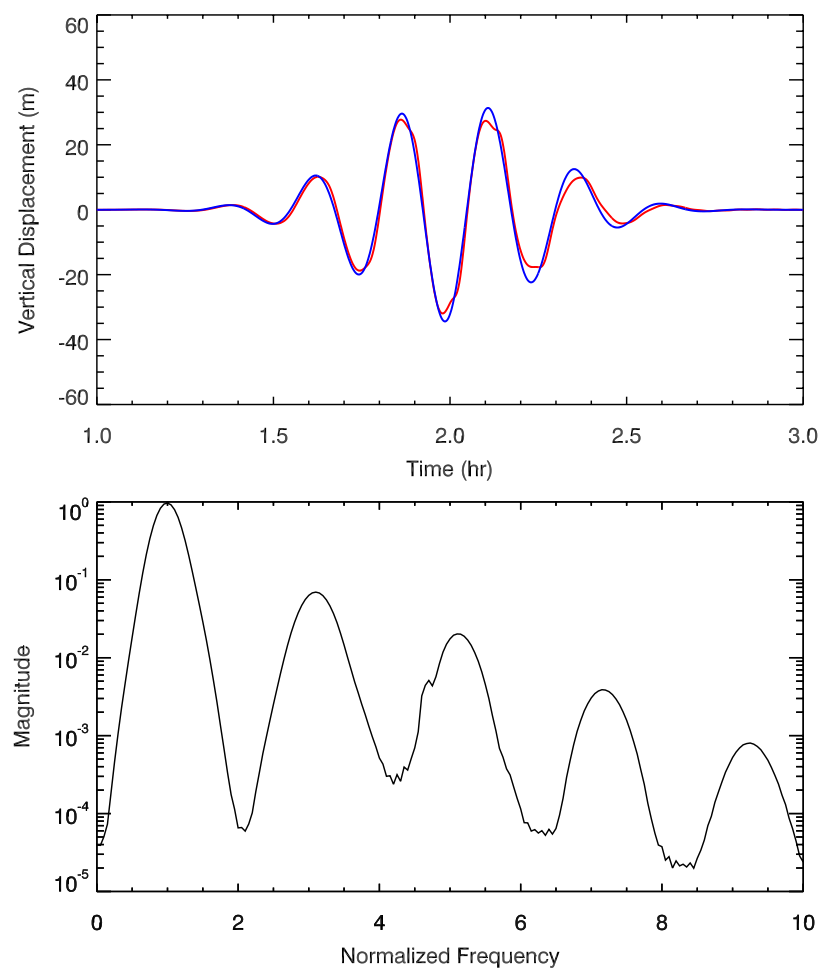

Fig. 4. As in Fig. 1, but for the SPB response to a gravity wave packet defined by Eq. (12).

The findings discussed above have ramifications for SPB measurements of gravity waves and the retrieval of important wave parameters, such as momentum flux. This issue is discussed further in the next section.

\section{Simulations and retrieval of gravity wave parameters}

Boccara et al. (2008) described a methodology by which SPB observations made during the Vorcore campaign could be analyzed to obtain gravity wave characteristics. To test the methodology, a series of Monte Carlo-type simulations were made that mimicked the SPB observations of GW-induced perturbations in pressure and horizontal balloon displacement. It was assumed that waves occurred in packets and a wavelet analysis technique was used to detect the packets in space and time and so to estimate the wave parameters.

In the Boccara et al. (2008) simulations, waves were allowed to propagate in random directions in the horizontal, but it was assumed that all waves propagated energy and momentum upward. Using the associated errors in the measured meteorological parameters and by repeating the simulations many times they were able to estimate the uncertainties and biases in the retrieved GW parameters, such as momentum flux. Briefly, it was found that, the horizontal direction of wave propagation was accurately retrieved but that momentum fluxes were somewhat underestimated.
Here we make use of the techniques described in Sect. 3 above to accurately model the SPB displacements and repeat the Boccara et al. (2008) simulations, but with the measurement parameters and uncertainties appropriate to the Concordiasi campaign SPB observations. There were important differences between the Vorcore and Concordiasi observations which make the later measurements of wave fluxes more accurate:

- Observations were made at $30 \mathrm{~s}$ intervals in Concordiasi, but only at $15 \mathrm{~min}$ intervals in Vorcore. Hence, in Concordiasi the full spectrum of GW motions from the between the Brunt-Väisälä ( $5 \mathrm{~min}$ ) and inertial $(\sim 13 \mathrm{~h})$ periods could be studied, whereas in Vorcore the measurements were restricted to periods greater than $1 \mathrm{~h}$ (Hertzog et al., 2008).

- More sensitive GPS measurements were available on the SPB during Concordiasi than in Vorcore, with Table 3 summarizing the instrumental uncertainties. Most importantly, it was possible to measure directly the vertical displacement of the balloons with an accuracy improved by a factor of 10 compared to the previous campaign. Having direct measurements of vertical displacement means that momentum fluxes can be derived without using the indirect and less accurate method used in Boccara et al. (2008), as discussed below.

\subsection{Simulations}

To test our retrievals of gravity wave parameters a large number of simulated SPB observations was made and then analyzed and the results compared with the original input parameters. Each simulation produced a notional 10-day time series with a basic $30 \mathrm{~s}$ time sample period. The balloons were assumed to drift eastward with a constant zonal wind speed of $10 \mathrm{~m} \mathrm{~s}^{-1}$ at a latitude of $60^{\circ} \mathrm{S}$, so that, without any wave perturbations, there was a steady change with time in the longitudinal position, but not in the latitudinal.

Time series were of the SPB observables: pressure $(\bar{p}+$ $\left.p_{T}^{\prime}\right)$, temperature $\left(\bar{T}+T_{T}^{\prime}\right)$, position in terms of longitude and latitude $\left(\bar{x}+x^{\prime}, y^{\prime}\right)$ and vertical balloon displacement $\left(\zeta_{b}^{\prime}\right)$ were then synthesized. Here, an overbar indicates the ambient value while the primed value indicates the wave-induced perturbation. It should be noted that the pressure and temperature perturbations are a combination of the relevant wave perturbation and of the pressure and temperature changes due to the vertical displacement of the balloon in the presence of background gradients

$p_{T}^{\prime}=p^{\prime}+\frac{\mathrm{d} \bar{p}}{\mathrm{~d} z} \zeta_{b}^{\prime}$,
$T_{T}^{\prime}=T^{\prime}+\frac{\mathrm{d} \bar{T}}{\mathrm{~d} z} \zeta_{b}^{\prime}$. 
Table 3. SPB measurement uncertainties.

\begin{tabular}{lll}
\hline Parameter & Symbol & Uncertainty \\
\hline Zonal displacement & $\sigma_{x}$ & $0.75 \mathrm{~m}$ \\
Meridional displacement & $\sigma_{y}$ & $0.75 \mathrm{~m}$ \\
Vertical displacement & $\sigma_{z}$ & $1.5 \mathrm{~m}$ \\
Zonal velocity & $\sigma_{u}$ & $0.025 \mathrm{~m} \mathrm{~s}^{-1}$ \\
Meridional velocity & $\sigma_{\mathrm{v}}$ & $0.025 \mathrm{~m} \mathrm{~s}^{-1}$ \\
Pressure & $\sigma_{p}$ & $0.1 \mathrm{~Pa}$ \\
Temperature & $\sigma_{T}$ & $0.3 \mathrm{~K}$ \\
\hline
\end{tabular}

Wave packets for a general wave parameter $\psi^{\prime}$ were derived with the form

$\psi^{\prime}=\operatorname{Re}\left(\underline{\psi} e^{-\frac{t^{2}}{2 t g}} e^{i(k x+l y+m z-\hat{\omega} t)}\right)$,

where Re means the real part; $\psi$ is the complex wave perturbation amplitude derived from the gravity wave polarization relationships; $k, l$ and $m$ are the zonal, meridional and vertical wavenumber, respectively. The basic methodology for each simulation is

1. First, choose $\hat{\omega}$ from a uniform random distribution in the range $|f|<\hat{\omega}<N$.

2. Then choose the intrinsic phase speed, $\hat{c}$, and direction of propagation, $\theta$ (counterclockwise from east), from uniform random distributions in the ranges $0 \leq \hat{c} \leq$ $100 \mathrm{~m} \mathrm{~s}^{-1}$ and $0 \leq \theta<360^{\circ}$. The zonal and meridional wavenumbers are then derived from $k=k_{\mathrm{h}} \cos \theta$ and $l=k_{\mathrm{h}} \sin \theta$, where $k_{\mathrm{h}}=\hat{\omega} / \hat{c}$.

3. The vertical wavenumber is derived from the dispersion equation

$|m|=\sqrt{\frac{N^{2}-\hat{\omega}^{2}}{\hat{\omega}^{2}-f^{2}} k_{\mathrm{h}}^{2}-\frac{1}{4 H^{2}}}$,

where $H$ is the density scale height. In contrast to Boccara et al. (2008), the sign of $m$ is set randomly, so that $-|m|(+|m|)$ means a wave with an upward (downward) group velocity.

4. The complex wave amplitudes are then computed. In order to make the simulations as realistic as possible, the horizontal perturbation velocity aligned along the direction of propagation, $u_{\|}^{\prime}$, was first derived at the appropriate $\hat{\omega}$ based on the mean horizontal wind spectrum derived from the actual SPB observations. Other wave parameters, $u^{\prime}, v^{\prime}, w^{\prime}, p^{\prime}, T^{\prime}$ are then derived from the GW polarization relations (Fritts and Alexander, 2003, 2012).

5. The vertical displacement of the SPB, $\zeta_{b}^{\prime}$, is then computed from the wave vertical displacement, $\zeta^{\prime}=$ $i w^{\prime} / \hat{\omega}$, using either of the methods discussed in Sect. 3.
6. Finally, the total pressure and temperature values were computed from Eqs. (16) and (17) and time series of all observables computed and saved for later analysis.

The above procedure was repeated 1000 times so that the retrievals of wave parameters could be tested over the complete spectrum of wave frequencies and propagation directions.

\subsection{Retrievals}

The formulae used to retrieve the wave characteristics from the balloon observations are based on those of Boccara et al. (2008). However, their work only dealt with hydrostatic waves and used only pressure measurements to infer the balloon vertical displacements. The two improvements achieved during the recent Concordiasi campaign (i.e., higher sampling rate and better precision of GPS vertical positions) enable us to relax these constraints, and extend the previous formulae. In the following description of the wave characteristics retrieval algorithm, we focus on its novel features and only briefly mention those that have not changed, for which Boccara et al. (2008) should be consulted.

As stated previously, the balloon observables are the 3D position, pressure $(p)$, and temperature $(T)$. At first, the zonal and meridional velocities ( $u$ and $v$, respectively) are computed by centered finite differences from the horizontal positions. The density $(\rho)$ is obtained using the perfect gas law:

$\rho=\frac{p}{R_{\mathrm{a}} T}$.

A flight-mean density $(\bar{\rho})$ and pressure $(\bar{p})$ are computed, and the total pressure perturbation is obtained from the latter as $p_{T}^{\prime}=p-\bar{p}$. Similarly, the perturbations in zonal and meridional velocities ( $u^{\prime}$ and $v^{\prime}$, respectively) are obtained as departures from the flight mean values. The Eulerian pressure perturbation $\left(p^{\prime}\right)$ is then estimated from the total pressure perturbation:

$p^{\prime}=p_{T}^{\prime}+\bar{\rho} g \zeta_{b}^{\prime}$,

which is the reciprocal of Eq. (16) assuming hydrostatic equilibrium for the background atmosphere. Note here that the balloon vertical displacement $\left(\zeta_{b}^{\prime}\right)$ is simply the departure from the flight-mean altitude. In particular, no assumption is made at this stage about the balloon flying at constant density.

A complex Morlet wavelet transform (Torrence and Compo, 1998) is then applied to all time series $\left(u^{\prime}, v^{\prime}, \zeta_{b}^{\prime}\right.$, $\left.p_{T}^{\prime}, p^{\prime}\right)$. From now on, all the equations in this section refer to the complex amplitudes of the wavelet coefficients, which are denoted with a tilde over the perturbations (e.g., $\tilde{u}$ ). These coefficients correspond to the decomposition of the wave signals in small $\Delta \hat{\omega}-\Delta t$ blocks in the intrinsic frequency-time domain. The wavelet set of frequencies are chosen to match the range of gravity-wave intrinsic frequencies (i.e., from $|f|$ to $N$ ). 
As in Boccara et al. (2008), $\theta$ is determined as the angle for which the modulus of the horizontal wind perturbation projected on that direction is maximized. $\theta$ is thus found with a $180^{\circ}$ ambiguity, which is resolved later on.

The intrinsic phase speed in the wave direction of propagation is readily inferred from the polarization relation (e.g., Fritts and Alexander, 2012):

$\tilde{p}=\bar{\rho} \hat{c} \delta_{-} \tilde{u}_{\|}$,

where $\delta_{-}=1-f^{2} / \hat{\omega}^{2}$. Hence, $\hat{c}$ is estimated as

$\hat{c}=\frac{1}{\bar{\rho} \delta_{-}} \frac{\operatorname{Re}\left(\tilde{p} \tilde{u}_{\|}^{*}\right)}{\tilde{u}_{\|}^{2}}$,

where the $\tilde{u}_{\|}^{*}$ denotes the complex conjugate of $\tilde{u}_{\|}$.

To compute the wave momentum flux, we assume that the balloon vertical displacement is that of a perfect isopycnic tracer. As previously discussed, this will be a source of error when the balloon departs from this ideal behavior (i.e., when $\hat{\omega} \rightarrow N$ ). Yet this assumption enables us to relate the balloon vertical displacements to those of air parcels. In particular, the Lagrangian component of the pressure disturbance $\left(\frac{\mathrm{d} \bar{p}}{\mathrm{~d} z} \zeta_{b}^{\prime}\right)$ can then be related to the Eulerian value:

$\frac{\mathrm{d} \bar{p}}{\mathrm{~d} z} \tilde{\zeta}_{b}=\left(i m H-\frac{1}{2}\right)\left(1+\frac{\hat{\omega}^{2}}{N^{2}-\hat{\omega}^{2}}\right) \tilde{p}$.

This equation is obtained in the same manner and is equivalent to Eq. (9) in Boccara et al. (2008), but includes in the second bracket an additional term associated with nonhydrostatic waves. Similarly, we use the full non-hydrostatic polarization relation between the horizontal and vertical velocity disturbances:

$\tilde{w}=-\left(\frac{\hat{\omega}^{2}-f^{2}}{N^{2}-\hat{\omega}^{2}}\right) \frac{m+i / 2 H}{k_{h}} \tilde{u}_{\|}$

which, with the help of Eq. (24), enables us to relate the wave momentum flux from the balloon observables:

$\operatorname{Im}\left(\tilde{p}_{T} \tilde{u}_{\|}^{*}\right)=-\bar{\rho} H \frac{N^{2}}{\hat{\omega}} \operatorname{Re}\left(\tilde{u}_{\|}^{*} \tilde{w}\right)$,

where $\operatorname{Im}(z)$ stands for the imaginary part of $z$. Equation (26) turns out to be the same equation as the hydrostatic version of Boccara et al. (2008). We demand here that the momentum flux be positive, which may require a sign switch of $\tilde{u}_{\|}$ (i.e., a rotation of $\theta$ by $180^{\circ}$ ). In other words, at this stage of the analysis all the wave packets are assumed to propagate upward in the atmosphere.

The vertical wavenumber of the wave packets can be inferred from a combination of Eqs. (22) and (25):

$m=-\bar{\rho}^{2} \hat{c} \delta_{-}\left(\frac{N^{2}-\hat{\omega}^{2}}{\hat{\omega}}\right) \frac{\operatorname{Re}\left(\tilde{u}_{\|}^{*} \tilde{w}\right)}{\tilde{p}^{2}}$.
Note that, in agreement with the previous assumption on the wave vertical direction of propagation, $m<0$ here. The actual sign of $m$ is now determined as follows. First, expressing $\tilde{p}_{T}$ as a function of $\tilde{w}$ with the help of Eqs. (22), (24) and (25), one obtains

$\operatorname{Re}\left(\tilde{w} \tilde{p}_{T}^{*}\right)=-\bar{\rho}\left(\frac{m}{m^{2}+1 / 4 H^{2}}\right)\left(\frac{N^{2}-\hat{\omega}^{2}}{\hat{\omega}}\right) \tilde{w}^{2}$.

The sign of $\operatorname{Re}\left(\tilde{w} \tilde{p}_{T}^{*}\right)$ is thus the opposite to that of $m$. Because $\zeta_{b}^{\prime} \equiv H R$ for a perfect isopycnic balloon one obtains with the help of Eq. (5):

$\operatorname{Re}\left(\tilde{w} \tilde{p}_{T}^{*}\right)=\operatorname{Re}\left(-i \frac{g \hat{\omega}}{H N^{2}} \tilde{\zeta}_{b} \tilde{p}_{T}^{*}\right)$.

Hence, the sign of $m$ can be inferred from the balloon observables. If $m>0, \theta$ is rotated by $180^{\circ}$, and the $\operatorname{sign}$ of $\operatorname{Re}\left(\tilde{u}_{\|}^{*} \tilde{w}\right)$ is reversed. This process fully resolves the initial $180^{\circ}$ ambiguity in $\theta$.

The horizontal wavenumber $k_{h}$ is then derived from Eq. (19), the gravity wave dispersion relation. Finally, the ground-based angular frequency $(\omega)$ is obtained from the Doppler-shift equation:

$\omega=\hat{\omega}+\bar{u} k_{h} \cos \theta+\bar{v} k_{h} \sin \theta$.

\subsection{Results}

Instrumental and wave propagation factors always impose limits on the extraction of GW parameters from observations (Alexander, 1998; Alexander and Barnet, 2007; Alexander et al., 2010). In principle, there are no limits on the range of $\mathrm{GW}$ frequencies or wavelengths that can be determined using SPBs of the type described here. However, there are likely to be difficulties in determining momentum fluxes for short period waves where a balloon departs from its EDS. Furthermore, the uncertainties that are inherent in the instruments carried on the SPB will set a noise floor, below which fluxes cannot be reliably determined. Similarly, the wavelet analysis itself will start to breakdown when packet amplitudes fall below some critical value. The procedures described above allow the limitations of the SPB momentum flux measurements and the uncertainties of other wave parameter to be explored.

In order to test the various factors that influence the accuracy of the SPB flux measurements, a series of preliminary investigations were conducted. Outcomes of trials that do not contain instrumental noise indicate the influence of the wavelet analysis technique and of the retrieval algorithm. Repeating the analysis of the same data set, but with noise now included, then shows the effects of instrumental noise. Results for one such comparison are illustrated in Fig. 5. Here a wave packet of the form

$u_{\|}^{\prime}=u_{o} e^{-t^{2} / 2 \hat{\tau}^{2}} \cos (\hat{\omega} t)$ 

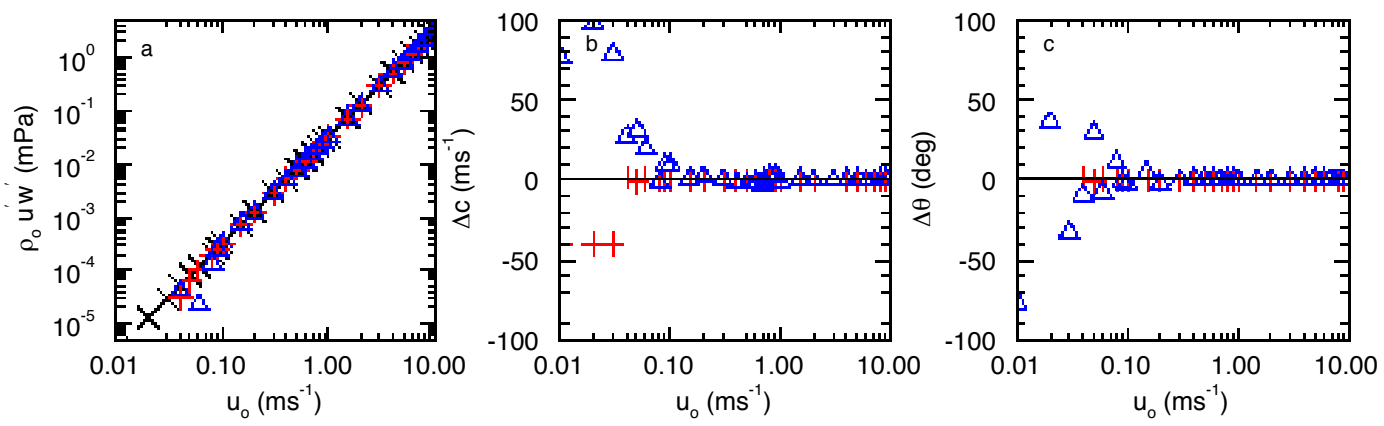

Fig. 5. (a) Values of momentum flux in mPa plotted as a function of wave packet amplitude, $u_{o}$. The $\times$ symbols represent the input values, the red + indicate the retrieved values without instrumental noise and the blue triangles show the retrieved values with noise. (b) is similar to (a), but where $\Delta c=\hat{c}_{\text {sim }}-\hat{c}_{\text {in }}$. (c) is similar to (a), but where $\Delta \theta=\theta_{\text {sim }}-\theta_{\text {in }}$.

was used. Other wave parameters were derived via the $\mathrm{GW}$ polarization relations as the packet amplitude, $u_{o}$, was changed systematically from 0.001 to $10 \mathrm{~m} \mathrm{~s}^{-1}$. In this example values of $\hat{\tau}=60 \mathrm{~min}, \hat{c}=40 \mathrm{~m} \mathrm{~s}^{-1}$ and $\theta=300^{\circ}$ were used, but the conclusions are quite general.

Figure 5a shows that $u^{\prime} w^{\prime}$ is determined well for values of $u_{o} \gtrsim 0.05 \mathrm{~m} \mathrm{~s}^{-1}$ for trials both with and without noise. In the case of $\hat{c}$ and of $\theta$ the same situation applies for the no noise case, but the effects of instrumental noise become noticeable for values of $u_{o} \lesssim 0.2 \mathrm{~m} \mathrm{~s}^{-1}$. Similar outcomes were found for other wave parameters, such as wavelength, which indicates that all wave parameters can be successfully retrieved if the velocity amplitudes are above a threshold of $u_{o} \sim 0.2 \mathrm{~m} \mathrm{~s}^{-1}$, although $u^{\prime} w^{\prime}$ can be reliably determined to lower values.

In the results discussed below 1000 simulations were used. Deriving and then retrieving data from this number of simulations is quite time consuming, so the analytic method was used to determine $\zeta_{b}^{\prime}$, since the results are similar to the more time-consuming numerical technique. Figures 6 and 7 show plots of retrievals of GW parameters from datasets that either include instrumental noise (lower panels) or no noise (upper panels). Results color coded in red and blue denote waves with $m<0$ and $m>0$, respectively.

Simulations of momentum flux show very good comparisons between the input and output values at all periods greater than $\sim 10-20 \mathrm{~min}$ (Fig. 6a, d). The effects of instrumental noise are minimal. However, it is clear that there are systematic differences between the input and retrieved values at short periods. To understand why, consider Eq. (26), which can be expressed using Eq. (15) from Boccara et al. (2008), as

$\operatorname{Re}\left(\tilde{u}_{\|}^{*} \tilde{w}\right)=-\frac{1}{\bar{\rho} g} \frac{(g / R+\partial \bar{T} / \partial z)}{\left(g / c_{p}+\partial \bar{T} / \partial z\right)} \operatorname{Im}\left(\tilde{p}_{T} \tilde{u}_{\|}^{*}\right)$,

which in turn can be expressed as

$$
\begin{aligned}
\operatorname{Re}\left(\tilde{u}_{\|}^{*} \tilde{w}\right) & =-\frac{1}{\bar{\rho} g} \frac{3 \omega_{\mathrm{B}}^{2}}{2 N^{2}} \operatorname{Im}\left(\tilde{p}_{T} \tilde{u}_{\| \mid}^{*}\right) \\
& =-\frac{1}{\bar{\rho} g|Z|_{\mathrm{EDS}}} \operatorname{Im}\left(\tilde{p}_{T} \tilde{u}_{\|}^{*}\right),
\end{aligned}
$$

where $|Z|_{\text {EDS }}$ was defined in Sect. 4. So the systematic deviations in retrieved flux at short periods mark the departure of the balloon off its EDS.

The retrievals of phase speed and direction are also excellent, especially in the non-noisy situations (Fig. 6b, c), but they show some systematic differences when wave frequencies are near $f$ and $N$, especially when instrumental noise is included (Fig. 6e, f). For $\theta$, when $\hat{\omega} \sim f$ the wind perturbation hodograph is almost circular, which makes the precise determination of direction of propagation more difficult. This accounts for the small spread in values of $\theta$ near $f$. While the changes in $\theta$ are small (no more than a few degrees), the variations in $\hat{c}$ are proportionately larger at both ends of the spectrum. Figure 7 shows that similar systematic deviations from input values are evident at short and long periods in other important wave parameters.

There are a number of reasons why the retrieved values may show a bias at both short and inertial periods. Firstly, the retrieval analysis assumes that the SBP is moving on an isopycnic surface, but the SPB departs significantly from its EDS at short periods, as illustrated in Fig. 3. In particular, it is the phase variations in $\underline{Z}$ that vary most rapidly with frequency for $N>\hat{\omega}>N / 2$, and produce the systematic bias.

A second, more subtle, effect is caused by the use of wave packets in the simulations. Packets described by Eq. (31) have a width in frequency space of $\Delta \omega \sim \hat{\omega}$. When either $\hat{\omega} \sim N$ or $\hat{\omega} \sim|f|$ the wave packets will project onto some wavelet coefficients associated with frequencies greater than $N$ or less than $|f|$. Furthermore, in this situation factors such as $\left(N^{2}-\hat{\omega}^{2}\right),\left(\hat{\omega}^{2}-f^{2}\right)$ or $\delta_{-}$, which appear in almost all expressions used to retrieve the wave parameters, reverse sign thereby accentuating the effect. Nevertheless, these "nongravity wave" coefficients are retained in the retrieval process 

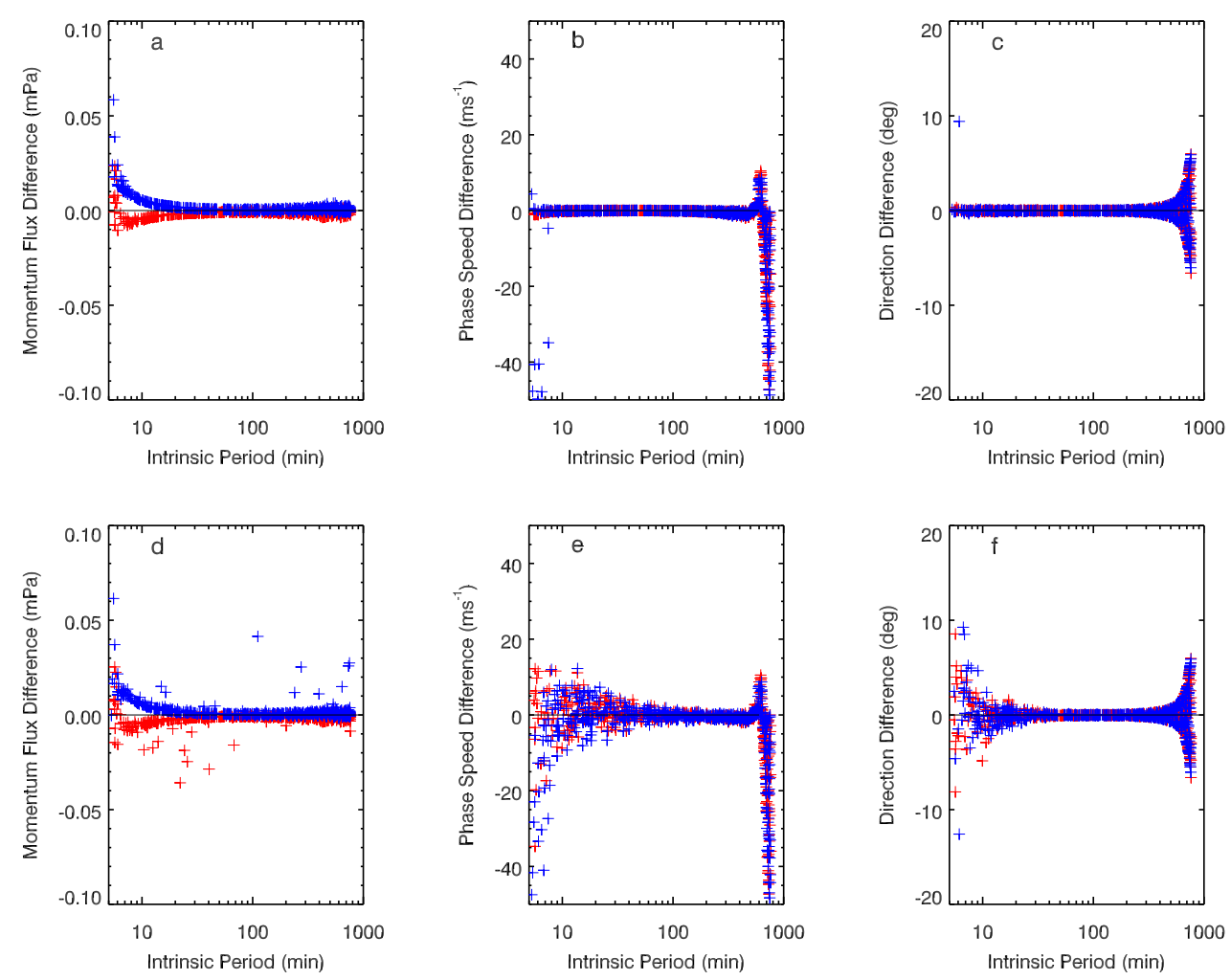

Fig. 6. Difference between computed and input values of momentum flux $\left(\rho_{o} u^{\prime} w_{\text {sim }}^{\prime}-\rho_{o} u^{\prime} w_{\text {in }}^{\prime}\right)$ as a function of intrinsic period for (a) without instrumental noise and (d) with noise. Panels (b) and (e) are similar to (a) and (d) where the only difference is between the computed and input intrinsic phase speeds $\left(\Delta c=\hat{c}_{\text {sim }}-\hat{c}_{\text {in }}\right)$. Panels (c) and (f) are similar to (a) and (d) where the only difference is between computed and input values of direction of propagation $\left(\Delta \theta=\theta_{\text {sim }}-\theta_{\text {in }}\right)$. In all panels, red (blue) values correspond to waves propagating energy upward (downward).

provided that the central frequency of the wave packet is located between $N$ and $|f|$. If they are discarded then a significant fraction of the wave momentum flux is lost.

Another factor in the degradation of performance near $N$ is the effect of instrumental noise (e.g., Fig. 6e) acting in concert with the change in wave amplitude with frequency in the simulations. As noted in item 4 in Sect. 5.1 above, the starting value of $u_{\|}$was derived from the observed spectrum of horizontal kinetic energy, which scales as $\sim \hat{\omega}^{-2}$. Hence, $u_{\|}$ is smaller at higher frequencies for shorter periods. Furthermore, the KE spectrum itself was derived from the average over all flights, which means that wave amplitudes for specific wave packets at a given frequency are probably underestimated, and are therefore more likely to be noisier than they would be in practice. A simple test in which the wave amplitudes input into the retrieval process were increased by a factor of 3 confirmed the latter hypothesis. It showed that the random variations at short periods evident in, say, Fig. 6e had almost disappeared.

Finally, it is stressed that the important momentum flux parameter is the one least influenced by noise. This supports the simulations shown in Fig. 5a, where values of $u^{\prime} w^{\prime}$ are recovered well down to small values of $u_{o}$. Momentum flux and wave propagation direction are also the two parameters that do not contain frequency dependent terms such as $\delta_{-}$, which explains the retrieval of these parameters over a wider frequency range.

Table 4 summarizes the statistics of the retrievals of important wave parameters. Except for the intrinsic and groundbased period ratios, the results for the whole wave spectrum and the more restricted frequency range $N / 2 \gtrsim \hat{\omega} \gtrsim 1.5 f$ are included. For the reasons discussed above, it is the latter frequency range that provides the more realistic results. For the wave periods the median values of the retrieved to input values are included as well as the mean values. For the intrinsic period the median and means are identical and show that the recovered values slightly underestimate the true values. The mean values of the ground-based periods are biased by some outliers, and the median values give a more accurate indiction of the accuracy of the retrieved values. Overall, the wave parameters are well recovered.

All the results just discussed have been obtained with time series containing a single gravity-wave packet. In the atmosphere, however, multiple sources acting at different times may simultaneously produce a number of wave packets in the volume sampled by SPB. Boccara et al. (2008) studied 

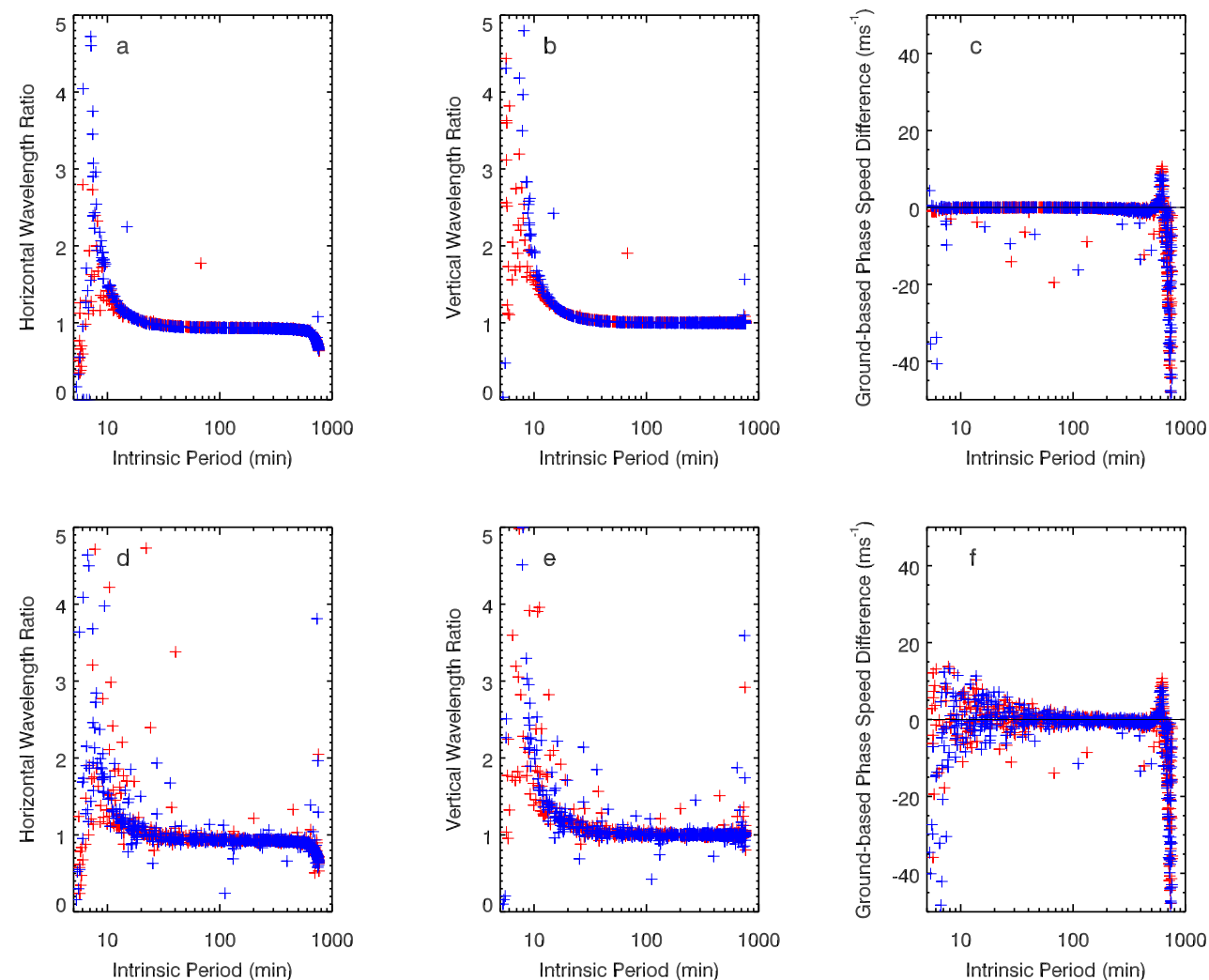

Fig. 7. As in Fig. 6, but for (a) and (d) the ratio of simulated to input horizontal wavelength $\left(\left(\lambda_{h}\right)_{\operatorname{sim}} /\left(\lambda_{h}\right)_{\text {in }}\right)$. (b) and (e) for vertical wavelength ratio $\left(\left(\lambda_{z}\right)_{\operatorname{sim}} /\left(\lambda_{z}\right)_{\text {in }}\right)$. (c) and (f) difference between simulated and input ground-based horizontal phase speed $\left(c g_{\text {sim }}-c g_{\text {in }}\right)$.

Table 4. Mean values of simulated parameters and their standard deviations. Here, $\Delta \hat{c}, \Delta \theta, \Delta\left(\rho_{o} u^{\prime} w^{\prime}\right)$ and $\Delta c_{\mathrm{g}}$ are the differences between the respective simulated and input values. The other quantities are the ratios of the simulated to input values. The $\hat{\tau}$ ratio denotes the ratio of the retrieved intrinsic wave period to the input value and the $\tau_{\mathrm{g}}$ ratio is the ratio of the retrieved to input ground-based period. Figures in brackets are median values. The statistics are provided for the full GW frequency range $(N>\hat{\omega}>f)$, unless otherwise stated, and both up and down going waves are included in the averages.

\begin{tabular}{lllll}
\hline Parameter & $\begin{array}{l}\text { Without noise } \\
\text { mean }\end{array}$ & SD & $\begin{array}{l}\text { With noise } \\
\text { mean }\end{array}$ & SD \\
\hline$\Delta \hat{c}\left(\mathrm{~m} \mathrm{~s}^{-1}\right)$ & -3.6 & 11.1 & -3.7 & 12.2 \\
$\Delta \hat{c}\left(\mathrm{~m} \mathrm{~s}^{-1}\right)(N / 2>\hat{\omega}>1.5 f)$ & -0.22 & 0.35 & 0.01 & 2.1 \\
$\Delta \theta(\mathrm{deg})$ & -0.73 & 13.8 & -2.8 & 22.4 \\
$\Delta \theta(\mathrm{deg})(N / 2>\hat{\omega}>1.5 f)$ & 0.00 & 0.2 & -0.82 & 12.1 \\
$\Delta\left(\rho_{o} u_{\|}^{\prime} w^{\prime}\right)(\mathrm{mPa})$ & $6 \times 10^{-4}$ & $5 \times 10^{-3}$ & $2 \times 10^{-4}$ & $7 \times 10^{-3}$ \\
$\Delta\left(\rho_{o} u_{\|}^{\prime} w^{\prime}\right)(\mathrm{mPa})(N / 2>\hat{\omega}>1.5 f)$ & $-5 \times 10^{-5}$ & $1 \times 10^{-3}$ & $-1 \times 10^{-4}$ & $4 \times 10^{-3}$ \\
$\Delta c_{\mathrm{g}}\left(\mathrm{m} \mathrm{s}^{-1}\right)$ & -3.8 & 11.3 & -4.0 & 12.2 \\
$\Delta c_{\mathrm{g}}\left(\mathrm{m} \mathrm{s}^{-1}\right)(N / 2>\hat{\omega}>1.5 f)$ & -0.43 & 1.5 & -0.12 & 2.5 \\
$\lambda_{\mathrm{h}}$ ratio & 2.2 & 40 & 1.1 & 3.6 \\
$\lambda_{\mathrm{h}}$ ratio $(N / 2>\hat{\omega}>1.5 f)$ & 0.97 & 0.11 & 1.1 & 2.0 \\
$\lambda_{z}$ ratio & 2.8 & 45 & 3.3 & 96 \\
$\lambda_{z}$ ratio $(N / 2>\hat{\omega}>1.5 f)$ & 1.05 & 0.12 & 1.07 & 12.3 \\
$\hat{\tau}$ ratio & $0.93(0.93)$ & 0.04 & $0.93(0.93)$ & 0.09 \\
$\tau_{\mathrm{g}}$ ratio & $1.48(0.93)$ & 15 & $1.44(0.93)$ & 10 \\
$\%$ of momentum flux in right direction & 98 & & 97 & \\
\hline
\end{tabular}


how the superposition of wave packets could change the performance of their retrieval algorithm. They noted first that the wavelet analysis used to retrieve wave parameters is well suited to separate wave packets that occur at the same time provided their respective central frequencies are sufficiently distinct. However, when superposition in the time-frequency space does occur, Boccara et al. (2008) noted a slight degradation of their retrieval, for example, gravity-wave momentum fluxes could be underestimated by $\sim 20 \%$ when 10-day time series include 10 randomly chosen wave packets. Still, it is difficult to know precisely how many wave packets do occur within any given time interval in the real atmosphere. The number will vary due to many factors, including the distance from the source(s) and the dispersive characteristics of gravity waves contributing to separate wave packets according to their frequency (e.g., Prusa et al., 1996). Therefore, the multiple wave-packet experiments were not repeated, and we assume that the associated uncertainty in the retrieved wave parameters is negligible compared to uncertainties in current gravity-wave drag parameterization schemes.

\section{Conclusions}

Superpressure balloons provide the only direct way to measure, over wide geographic regions, momentum fluxes and other important wave parameters in terms of intrinsic frequency and phase speed. These measurements help constrain gravity-wave drag parameterization schemes, notably the distribution of momentum flux as a function of the 2D horizontal phase speed. Building on the work of Nastrom (1980) and others, we analyze the response of an SPB to vertical displacements induced by gravity waves. Using the known uncertainties of the various instruments carried on the latest versions of SPB developed by CNES, we estimate the accuracy to which fluxes and other important wave parameters can be measured as a function of wave amplitude. The analysis is particularly focussed on SPB operating in the stratosphere.

Both numerical and quasi-analytic techniques are used, with the analytic technique giving particular insight into the SPB response as a function of wave frequency. It is shown that the response is well behaved for intrinsic wave frequencies lower than about $N / 2$. At low frequencies the ratio of the balloon vertical displacement to the wave displacement has a limiting value determined solely by atmospheric temperature and its gradient. Numerically the value is about 0.25 for conditions in the Antarctic springtime stratosphere. At frequencies higher than $\sim N / 2$, the balloon starts to depart significantly from its isopycnic surface or EDS.

Following Boccara et al. (2008) a statistical analysis of the simulated response of $12 \mathrm{~m}$ diameter SPB to gravity wave packets propagating in the Antarctic stratosphere is used to show that momentum flux is measured with high accuracy for $\hat{\omega} \lesssim N / 2$, as is the direction of wave propagation.
Momentum fluxes can be accurately measured down to values of about $10^{-4} \mathrm{mPa}$ (Fig. 5a). As newer instruments are installed, including more accurate GPS measurements of displacement, reductions in this noise floor are possible. Other wave parameters such as intrinsic phase speed and horizontal and vertical wavelengths are also recovered with good accuracy, although the optimum frequency range is $N / 2 \gtrsim \hat{\omega} \gtrsim$ $1.5 f$ due to factors that complicate the retrieval process when $\hat{\omega} \sim f$.

An important outcome is that the retrieval process is independent of the vertical direction of wave propagation propagation. This means that it will be possible to derive the net momentum flux when the analysis is applied to real data, such as that acquired during the 2010 Concordiasi campaign.

Acknowledgements. RAV gratefully acknowledges support from LMD when visiting AH during the course of this research. He also thanks Chris Pietsch for contributions to the numerical solution of SPB motions. AH would like to thank Francis Dalaudier for insightful comments on an earlier version of the manuscript, as well as Étienne Vignon who contributed to the derivation of retrieval formulae during his internship at LMD. We acknowledge CNES support for long-duration balloon activities. The referees are thanked for their helpful comments.

Edited by: A. Stoffelen

\section{References}

Alexander, M. J.: Interpretations of observed climatological patterns in stratospheric gravity wave variance, J. Geophys. Res., 103, 8627-8640, 1998.

Alexander, M. J. and Barnet, C.: Using satellite observations to constrain parameterizations of gravity wave effects for global models, J. Atmos. Sci., 64, 1652-1665, 2007.

Alexander, M. J., Geller, M., McLandress, C., Polavarapu, S., Preusse, P., Sassi, F., Sato, K., Ern, M., Hertzog, A., Kawatani, Y., Pulido, M., Shaw, T., Sigmond, M., Vincent, R., and Watanabe, S.: Recent developments in gravity wave effects in climate models and the global distribution of gravity wave momentum flux from observations and models, Q. J. Roy. Meteorol. Soc., 136, 1103-1124, 2010.

Beres, J. H.: Gravity wave generation by a three-dimensional thermal forcing, J. Atmos. Sci., 61, 1805-1815, 2004.

Boccara, G., Hertzog, A., Vincent, R. A., and Vial, F.: Estimation of gravity-wave momentum fluxes and phase speeds from quasiLagrangian stratospheric balloon flights. 1: Theory and simulations, J. Atmos. Sci., 65, 3042-3055, 2008.

Eckermann, S. D., Gibson-Wilde, D. E., and Bacmeister, J. T.: Gravity wave perturbations of minor constituents: A parcel advection methodology, J. Atmos. Sci., 55, 3521-3539, 1998.

Fritts, D. C. and Alexander, M. J.: Gravity wave dynamics and effects in the middle atmosphere, Rev. Geophys., 41, 1003, doi:10.1029/2001RG000106, 2003.

Fritts, D. C. and Alexander, M. J.: Correction to "Gravity wave dynamics and effects in the middle atmosphere", Rev. Geophys., 50, RG3004, doi:10.1029/2012RG000409, 2012. 
Grise, K. M., Thompson, D. W. J., and Birner, T.: A global survey of static stability in the stratosphere and upper troposphere, J. Climate, 23, 2275-2292, 2010.

Hertzog, A., Cocquerez, P., Basdevant, C., Boccara, G., Bordereau, J., Brioit, B., Cardonne, A., Guilbo, R., Ravissot, A., Schmitt, E., Valdivia, J.-N., Venel, S., and Vial, F.: Stratéole/Vorcore-Longduration superpressure balloons to study the Antarctic lower stratosphere during the 2005 winter, J. Atmos. Oceanic Tech., 24, 2048-2061, 2007.

Hertzog, A., Boccara, G., Vincent, R. A., Vial, F., and Cocquerez, P.: Estimation of gravity-wave momentum fluxes and phase speeds from quasi-lagrangian stratospheric balloon flights. 2: Results from the Vorcore campaign in Antarctica, J. Atmos. Sci., 65, 3056-3070, 2008.

Hines, C. O.: Internal atmospheric gravity waves at ionospheric heights, Can. J. Phys., 38, 1441-1481, 1960.

Jewtoukoff, V., Plougonven, R., and Hertzog, A.: Gravity waves generated by deep tropical convection: estimates from balloon observations and mesoscale simulations, J. Geophys. Res., 118, 9690-9707, doi:10.1002/jgrd.50781, 2013.

Lane, T. P. and Moncrieff, M. W.: Stratospheric gravity waves generated by multiscale tropical convection, J. Atmos. Sci., 65, 2598-2614, 2008.

Massman, W. J.: On the nature of vertical oscillations of constant volume balloons, J. Applied Meteor., 17, 1351-1356, 1978.

Massman, W. J.: An investigation of gravity waves on a global scale using TWERLE data, J. Geophys. Res., 86, 4072-4082, 1981.

Nastrom, G. D.: The response of superpressure balloons to gravity waves, J. Applied Meteor., 19, 1013-1019, 1980.

Piani, C., Durran, D., Alexander, M. J., and Holton, J. R.: A numerical study of three-dimensional gravity waves triggered by deep tropical convection and their role in the dynamics of the QBO, J. Atmos. Sci., 57, 3689-3702, 2000.
Press, W. H., Teukolsky, S. A., Vetterling, W. T., and Flannery, B. P.: Numerical Recipes in C: The Art of Scientific Computing, Cambridge University Press, 2nd Edn., 1992.

Preusse, P., Eckermann, S. D., and Ern, M.: Transparency of the atmosphere to short horizontal wavelength gravity waves, J. Geophys. Res., 113, D24104, doi:10.1029/2007JD009682, 2008.

Prusa, J. M., Smolarkiewicz, P. K., and Garcia, R. R.: Propagation and breaking at high altitudes of gravity waves excited by tropospheric forcing, J. Atmos. Sci., 53, 2186-2216, 1996.

Rabier, F., Bouchard, A., Brun, E., Doerenbecher, A., Guedj, S., Guidard, V., Karbou, F., Peuch, V.-H., El Amraoui, L., Puech, D., Genthon, C., Picard, G., Town, M., Hertzog, A., Vial, F., Cocquerez, P., Cohn, S. A., Hock, T., Fox, J., Cole, H., Parsons, D., Powers, J., Romberg, K., VanAndel, J., Deshler, T., Mercer, J., Haase, J. S., Avallone, L., Kalnajs, L., Mechoso, C. R., Tangborn, A., Pellegrini, A., Frenot, Y., Thepaut, J.-N., McNally, A., Balsamo, G., and Steinle, P.: The CONCORDIASI project in Antarctica, B. Am. Meteorol. Soc, 91, 69-86, 2010.

Torrence, C. and Compo, G. P.: A practical guide to wavelet analysis, B. Am. Meteorol. Soc, 79, 61-78, 1998.

TWERLE Team: The TWERL Experiment, B. Am. Meteorol. Soc., 58, 930-948, 1977.

Vincent, R. A., Hertzog, A., Boccara, G., and Vial, F.: Balloonborne measurements of gravity-wave momentum fluxes in the polar stratosphere, Geophys. Res. Lett., 34, L19804, doi:10.1029/2007GL031072, 2007.

Walterscheid, R. L., Galinas, L. J., Mechoso, C. R., and Schubert, G.: Evaluation of momentum and sensible heat fluxes in constant density coordinates: Application to superpressure balloon data during the VORCORE campaign, J. Geophys. Res., 117, D09105, doi:10.1029/2011jd016731, 2012. 